Review Article

\title{
Management of carcinoid syndrome
}

\author{
Asha Saini and Jonathan Waxman
}

Department of Clinical Oncology, Royal Postgraduate Medical School, Hammersmith Hospital, Du Cane Road, London W12 ONN, UK

\section{Introduction}

Carcinoid tumours are uncommon neoplasms with a reported incidence of 1.5 per 100000 population. Although they can develop in any organ derived from primitive endoderm, they occur most frequently in the appendix. Primary carcinoid tumours can be classified according to their site of origin. Although this classification into fore-gut, mid-gut or hind-gut tumours is of no prognostic significance, it is of clinical value in that the classical features of malignant carcinoid syndrome are much more likely in metastatic disease originating from a mid-gut primary tumour. These features, which include cutaneous flushing, diarrhoea and bronchospasm, result from the secretory products of this neuroendocrine tumour which include 5-hydroxytryptamine, kallikrein, tachykinins, substance $P$, prostaglandins and catecholamines. ${ }^{1,2}$ Carcinoid tumours of fore-gut origin tend to have different neuro-endocrine products, secreting histamine and 5-hydroxytryptophan.

5-Hydroxytryptamine (5-HT) is the cause of carcinoid diarrhoea and flushing may be related to either prostaglandin or tachykinin production. ${ }^{3}$

Patients with malignant carcinoid syndrome have a remarkably good prognosis even though the majority have liver metastases at presentation. Moertel reported a median survival of 38 months with a range extending to 15 years. ${ }^{4}$

In the Hammersmith Hospital series of 68 patients, $48 \%$ survived 5 years and $18 \% 10$ years. ${ }^{5}$ Prognosis depends upon the site of origin of the tumour, levels of secreted products, weight loss and tumour size. Patients with daily urinary 5-hydroxyindole acetic acid (5-HIAA) excretion greater than $1000 \mu \mathrm{mol}$ and with weight loss greater than $8 \mathrm{~kg}$ have been shown to have a poor prognosis. ${ }^{5}$ The likelihood of metastasis relates to the size of the primary tumour. Less than $2 \%$ of patients with

Correspondence: J. Waxman, M.D., B.Sc., F.R.C.P. Received: 10 January 1991 tumours under $1 \mathrm{~cm}$ in size have hepatic metastases but when the primary is over $2 \mathrm{~cm}$, almost $100 \%$ have metastatic disease.

\section{Therapeutic options}

The striking feature of carcinoid tumours is their relatively benign course and this defines therapy, for although tumour load may not be significantly changed by treatment, symptoms can be eased and quality of life greatly improved. In the last decadeo there have been significant changes in the way that carcinoid syndrome is managed and this relates to our understanding of the biochemical processes that lead to symptoms. From this understanding new treatments have developed.

\section{Chemotherapy for carcinoid tumours}

Cytotoxic chemotherapy is relatively ineffective in the treatment of carcinoid tumours. 5-Fluorouracil, streptozotocin, doxorubicin and cyclophosphamide are amongst the most effective single agents with response rates ranging up to $33 \%{ }^{4,6}$ These responses are generally for a short period of time and there is no advantage to combination chemotherapy. In one study, 5fluorouracil, adriamycin, cyclophosphamide and streptozotocin were combined and led to responses in $31 \%$ of the treated patients which was for a median duration of 5 months ${ }^{7}$ offering no advantage over single agent therapy. Newer agents developed in the last decade have been found to have little effect. ${ }^{8}$

\section{Interventional radiology}

Hepatic artery embolization in carcinoid syndrome was first reported in $1977 . .^{9}$ This technique involves selective cannulation of the hepatic artery and injection of embolic materials. In a recent series of 
24 patients treated in this manner at the Hammersmith Hospital there was sustained symptom relief in the majority, which had a median duration of 10 months. $^{5}$

Embolization may be repeated and confer further benefit. It may have complications and can result in the overwhelming release of vasoactive substances leading to circulatory instability and the precipitation of an acute carcinoid crisis. There is also the risk of infection within the necrotic embolized tissue and septicaemia. Hepatic enzymes usually increase, returning to pretreatment levels after approximately one week. This procedure is associated with a significant mortality and $3 \%$ of patients treated may die.

Although there is excellent palliation of symptoms, including the relief of hepatic pain, embolization has not been shown to confer a survival advantage. Because of the lack of survival advantage, morbidity and mortality, this approach should not be included amongst first line therapeutic options.

\section{Interferons}

The efficacy of recombinant alpha interferon in carcinoid syndrome has been demonstrated in several small series. An early study reported objective tumour response rates in $48 \%$ of patients. ${ }^{10}$ More recently, the effects of interferon were evaluated in 13 patients previously untreated. Human leucocyte interferon was given subcutaneously at doses of 3-6 million units for 5 days each week. All patients were treated for 12 months. Objective tumour responses, as measured by $50 \%$ or greater reduction in urinary 5-HIAA excretion or $\geqslant 50 \%$ reduction in the size of hepatic metastases occurred in $15 \%$. Stable disease was seen in $78 \%$ and there was one case of progression. Although these changes were associated with clear symptomatic improvement, the initial positive effect on diarrhoea waned with time. ${ }^{11}$

The high incidence of stable disease might suggest that interferon has a cytostatic effect. In this condition the slow progression of untreated disease makes the significance of this finding difficult to assess. However, in a randomized study the effects of human leucocyte interferon have been compared with streptozotocin and 5-fluorouracil and the superiority of interferon was demonstrated. ${ }^{12}$

A combined approach with removal of the primary tumour at laparotomy, followed by hepatic artery embolization and 12 months' treatment with interferon has been reported to produce an objective tumour response rate of $85 \%$ at 12 months, compared with $40 \%$ when interferon was given alone. ${ }^{13}$ Despite these findings it remains contentious whether interferon has any effect on tumour bulk, and disease progression.

\section{The pharmacological control of symptoms}

Drugs which block the synthesis, release or peripheral actions of circulating tumour products are appropriate first line agents. Their use remains empirical in view of the differing profiles of active substances released from any given tumour, but a classification based on their modes of action is helpful.

1. Inhibitors of 5-HT synthesis Amongst this group of agents, parachlorphenylalanine and chlorpromazine are included. Parachlorphenylalanine is an inhibitor of tryptophan hydroxylase, preventing the conversion of tryptophan to 5-HT. It relieves flushing and diarrhoea in $50-60 \%$ of cases and reduces urinary 5-HIAA excretion in up to $80 \%$ of cases. Its use is limited by a significant incidence of psychiatric symptoms including confusion and depression. Chlorpromazine may cause significant sedation and its use is of little value in the treatment of carcinoid syndrome.

2. Peripheral 5-HT antagonists Cyproheptadine, ketanserin, methysergide and ondansetron are included within this group of agents. Cyproheptadine at a dose of $4 \mathrm{mg}$ thrice daily led to an improvement in flushing in $47 \%$ and of diarrhoea in $60 \%$ of patients. The mean duration of symptom response was 8 months and side effects were minimal. ${ }^{3}$ Ketanserin is known to cause specific blockade of 5-HT2 receptors as well as $\mathrm{H} 1$ and alpha adrenoceptors. It controls flushing in about $50 \%$ of patients but only one fifth find relief from diarrhoea.

3. Antihistamines and other agents Combined $\mathrm{H} 1$ and $\mathrm{H} 2$ blockade using chlorpheniramine and cimetidine may be used in cases presenting atypically with urticarial skin rashes occurring in association with foregut primary carcinoids. When catecholamine synthesis is suspected the use of phenoxybenzamine may produce useful relief of symptoms. Aprotinin inhibits kinin production, and may sometimes be effective.

4. Inhibitors of 5-HT release Many clinicians now consider somatostatin analogues to be the treatment of first choice for patients with carcinoid syndrome. Somatostatin has been termed an 'endocrine bleach', inhibiting the release of neuropeptides and gut endocrine and exocrine function. It also inhibits other functions of the gut, decreasing mobility, absorption and blood flow. Early studies showed a reduction in symptoms caused by carcinoid lesions with treatment with octreotide, a long acting analogue of somatostatin. ${ }^{14}$ This long acting analogue has the advantage over the native hormone of a prolonged plasma 
half life of 90-120 minutes with a duration of action of 6-8 hours. The prolonged action of this octapeptide results from the presence of two Damino acids, which resist degradation by endogenous peptidases. Initial relief of symptoms has been reported in up to $80 \%$ of patients. ${ }^{14}$ The same study showed a nadir of 5-HIAA excretion at 1 month, with a median response duration of 12 months. Patients are taught to administer octreotide themselves and the only side effect is a slight discomfort at the injection site. There have been no reports of renal, hepatic, central nervous system or other toxicity, and the treatment is well tolerated. It is now known that with its prolonged use increasing dosages are required to maintain symptom control.

Somatostatin analogues are also used in the management of acute carcinoid crisis precipitated by surgery or radiological intervention, when ensu-

\section{References}

1. Conlon, J.M., Deacon, C.F., Richter, G., Stockmann, F. \& Creutzfeldt, W. Circulating tachykinins (Substance P, neurokinin $\mathbf{A}$, neuropeptide $\mathrm{K}$ ) and the carcinoid flush. Scand J Gastroenterol 1987, 22: 97-105.

2. Oberg, K., Norheim, I., Theodorsson, E., Ahlman, H., Lundqvist, G. \& Wide, L. The effects of octreotide on basal and stimulated hormone levels in patients with carcinoid syndrome. J Clin Endocrinol Metab 1989, 68: 796.

3. Vinik, A.I., Thompson, N., Eckhauser, F. \& Moattari, A.R. Clinical features of carcinoid syndrome and the use of somatostatin analogue in its management. Acta Oncol 1989, 28: Fasc 3

4. Moertel, C.G. Treatment of the carcinoid tumour and the malignant carcinoid syndrome. J Clin Oncol 1983, 1: 727-740.

5. Coupe, M., Levi, S., Ellis, M. et al. Therapy for symptoms in the carcinoid syndrome. $Q J$ Med 1989, 271: $1021-1036$.

6. Moertel, C.G. \& Hanley, J.A. Combination chemotherapy trials in metastatic carcinoid tumor and the malignant carcinoid syndrome. Cancer Clin Trials 1979, 2: 327-334.

7. Bukowski, R.M., Johnson, K.G., Peterson, R.F. et al. A phase II trial of combination chemotherapy in patients with metastatic carcinoid tumors. Cancer 1987, 60: 2891-2895.

8. Jodrell, D.I. \& Smith, I.E. Carboplatin in the treatment of metastatic carcinoid tumors and paraganglioma - a phase II study. Cancer Chemotherap Pharmacol 1990, 26: 62-64. ing cardiovascular instability can be rapidly reversed by intravenous administration of octreotide. $^{15}$

\section{Discussion}

Symptom control in metastatic carcinoid syndrome is of prime importance in view of the lack of effective treatment to reduce tumour bulk. The most effective therapy for this condition is somatostatin analogue treatment. It may be that new approaches using this compound in combination with biological response modifying agents such as the interferons may provide an advantage in terms of reduction in tumour volume as well as symptom relief. These studies are currently in progress.

9. Allison, D.J., Modlin, I.M. \& Jenkins, W.J. Treatment of carcinoid liver metastases by hepatic artery embolisation. Lancet 1979, ii: $1323-1325$.

10. Oberg, K., Norheim, I., Lind, E. et al. Treatment of malignant carcinoid tumours with human leucocyte interferon - long term results. Cancer Treat Rep 1986, 70: 1297-1304.

11. Nobin, A., Lindblom, A., Mansson, B. \& Sundberg, M. Interferon treatment in patients with malignant carcinoids. Acta Oncol 1989, 28: Fasc 3.

12. Oberg, K., Norheim, I. \& Alm, G. Treatment of malignant carcinoid tumours: a randomised controlled study of streptozotocin plus 5-Fu and human leucocyte interferon. Eur $J$ Cancer Clin Oncol 1989, 25: 1475-1479.

13. Hanssen, L.E., Schrumpf, E., Kolbenstvedt, A.N., Tausjo, J. \& Dolva, L.O. Recombinant alpha 2 interferon with or without hepatic artery embolisation in the treatment of midgut carcinoid tumours. Acta Oncol 1989, 28: Fasc 3.

14. Kvols, L.K., Moertels, C.G., O'Connell, M.J., Schute, A.J., Rudin, J. \& Hahn, R.G. Treatment of the malignant carcinoid syndrome, evaluation of a long acting somatostatin analogue. $N$ Engl J Med 1986, 35: 633-636.

15. Kvols, L.K., Martin, J.K., Marsh, H.M. \& Moertel, C.G. Carcinoid crisis: rapid reversal of life threatening hypotension with a somatostatin analogue. $N$ Engl J Med 1985, 313: 1229. 